\title{
Seek COVER: using a disease proxy to rapidly develop and validate a personalized risk calculator for COVID-19 outcomes in an international network
}

Ross D. Williams ${ }^{1 \dagger}$, Aniek F. Markus ${ }^{1 \dagger}$, Cynthia Yang ${ }^{1}$, Talita Duarte-Salles ${ }^{2}$, Scott L. DuVall ${ }^{3}$, Thomas Falconer ${ }^{4}$, Jitendra Jonnagaddala ${ }^{5}$, Chungsoo Kim ${ }^{6}$, Yeunsook Rho ${ }^{7}$, Andrew E. Williams ${ }^{8}$, Amanda Alberga Machado ${ }^{9}$, Min Ho An ${ }^{10}$, María Aragón ${ }^{2}$, Carlos Areia ${ }^{11}$, Edward Burn ${ }^{2,12}$, Young Hwa Choi ${ }^{13}$, lannis Drakos ${ }^{14}$, Maria Tereza Fernandes Abrahão ${ }^{15}$, Sergio Fernández-Bertolín², George Hripcsak, Benjamin Skov Kaas-Hansen ${ }^{16,17}$, Prasanna L. Kandukuri ${ }^{18}$, Jan A. Kors ${ }^{1}$, Kristin Kostka ${ }^{19}$, Siaw-Teng Liaw ${ }^{5}$, Kristine E. Lynch ${ }^{3}$, Gerardo Machnicki ${ }^{20}$, Michael E. Matheny ${ }^{21,22}$, Daniel Morales ${ }^{23}$, Fredrik Nyberg ${ }^{24}$, Rae Woong Park ${ }^{25}$, Albert Prats-Uribe ${ }^{12}$, Nicole Pratt ${ }^{26}$, Gowtham Rao ${ }^{27}$, Christian G. Reich ${ }^{19}$, Marcela Rivera ${ }^{28}$, Tom Seinen ${ }^{1}$, Azza Shoaibi ${ }^{27}$, Matthew E. Spotnitz ${ }^{4}$, Ewout W. Steyerberg ${ }^{29,30}$, Marc A. Suchard ${ }^{31}$, Seng Chan You ${ }^{25}$, Lin Zhang ${ }^{32,33}$, Lili Zhou ${ }^{18}$, Patrick B. Ryan ${ }^{27}$, Daniel Prieto-Alhambra ${ }^{12}$, Jenna M. Reps ${ }^{27 \dagger}$ and Peter R. Rijnbeek ${ }^{1 *+}$

\begin{abstract}
Background: We investigated whether we could use influenza data to develop prediction models for COVID-19 to increase the speed at which prediction models can reliably be developed and validated early in a pandemic. We developed COVID-19 Estimated Risk (COVER) scores that quantify a patient's risk of hospital admission with pneumonia (COVER-H), hospitalization with pneumonia requiring intensive services or death (COVER-I), or fatality (COVER-F) in the 30-days following COVID-19 diagnosis using historical data from patients with influenza or flu-like symptoms and tested this in COVID-19 patients.

Methods: We analyzed a federated network of electronic medical records and administrative claims data from 14 data sources and 6 countries containing data collected on or before 4/27/2020. We used a 2-step process to develop 3 scores using historical data from patients with influenza or flu-like symptoms any time prior to 2020. The first step was to create a data-driven model using LASSO regularized logistic regression, the covariates of which were used to develop aggregate covariates for the second step where the COVER scores were developed using a smaller set of features. These 3 COVER scores were then externally validated on patients with 1) influenza or flu-like symptoms and 2) confirmed or suspected COVID-19 diagnosis across 5 databases from South Korea, Spain, and the United States.
\end{abstract}

\footnotetext{
${ }^{*}$ Correspondence: p.rijnbeek@erasmusmc.nl

${ }^{\dagger}$ Ross D. Williams, Aniek F. Markus, Jenna M. Reps and Peter R. Rijnbeek contributed equally to this work.

${ }^{1}$ Department of Medical Informatics, Erasmus University Medical Center,

Doctor Molewaterplein, 403015, GD, Rotterdam, The Netherlands

Full list of author information is available at the end of the article
}

\section{$\triangle B M C$} permits use, sharing, adaptation, distribution and reproduction in any medium or format, as long as you give appropriate credit to the original author(s) and the source, provide a link to the Creative Commons licence, and indicate if changes were made. The images or other third party material in this article are included in the article's Creative Commons licence, unless indicated otherwise in a credit line to the material. If material is not included in the article's Creative Commons licence and your intended use is not permitted by statutory regulation or exceeds the permitted use, you will need to obtain permission directly from the copyright holder. To view a copy of this licence, visit http://creativecommons.org/licenses/by/4.0/. The Creative Commons Public Domain Dedication waiver (http://creativeco mmons.org/publicdomain/zero/1.0/) applies to the data made available in this article, unless otherwise stated in a credit line to the data. 
Outcomes included i) hospitalization with pneumonia, ii) hospitalization with pneumonia requiring intensive services or death, and iii) death in the 30 days after index date.

Results: Overall, 44,507 COVID-19 patients were included for model validation. We identified 7 predictors (history of cancer, chronic obstructive pulmonary disease, diabetes, heart disease, hypertension, hyperlipidemia, kidney disease) which combined with age and sex discriminated which patients would experience any of our three outcomes. The models achieved good performance in influenza and COVID-19 cohorts. For COVID-19 the AUC ranges were, COVERH: 0.69-0.81, COVER-I: 0.73-0.91, and COVER-F: 0.72-0.90. Calibration varied across the validations with some of the COVID-19 validations being less well calibrated than the influenza validations.

Conclusions: This research demonstrated the utility of using a proxy disease to develop a prediction model. The 3 COVER models with 9-predictors that were developed using influenza data perform well for COVID-19 patients for predicting hospitalization, intensive services, and fatality. The scores showed good discriminatory performance which transferred well to the COVID-19 population. There was some miscalibration in the COVID-19 validations, which is potentially due to the difference in symptom severity between the two diseases. A possible solution for this is to recalibrate the models in each location before use.

Keywords: Patient-level prediction modelling, COVID-19, Risk score

\section{Background}

In early 2020 the growing number of infections due to the coronavirus disease 2019 (COVID-19) resulted in unprecedented pressure on healthcare systems worldwide and caused many casualties at a global scale. Although the majority of people had uncomplicated or mild illness (81\%), some developed severe disease leading to hospitalization and oxygen support (15\%) or fatality $(4 \%)[1,2]$. This presented a challenge both in finding effective treatments as well as in identifying which patients were at high risk and as such would benefit from protective measures. The most common diagnosis in severe COVID-19 patients was pneumonia, other known complications included acute respiratory distress syndrome (ARDS), sepsis, or acute kidney injury (AKI) [1].

The WHO Risk Communication Guidance distinguished two categories of patients at high risk of severe disease: those older than 60 years and those with "underlying medical conditions", which is non-specific [3]. Using general criteria to assess the risk of poor outcomes is a crude risk discrimination mechanism as entire patient groupings are treated homogeneously ignoring individual differences. Prediction models can quantify a patient's individual risk and data-driven methods could help to identify risk factors that have been previously overlooked. However, a systematic review evaluating all available prediction models for COVID-19 [4] concluded that despite the large number of prediction models being developed for COVID-19, none were considered ready for clinical practice. These COVID-19 prediction models were criticized for i) being developed using small data samples, ii) lacking external validation, and iii) being poorly reported.

In this article, we describe a process of using a proxy disease to develop a prediction model for another disease. This can be used in situations where there is a data scarcity for the disease of interest. In this process a model is developed using big data from a proxy disease and then assessed in the target disease. This preserves all the target disease data for validation to provide a more robust and reliable assessment of model performance in the intended setting. This increases the evidence of the performance of a model in the target disease compared to if the same data had been used for development. We describe a use-case for this process using influenza data to develop a model in the early stages of the COVID-19 pandemic. It has been well documented that influenza and COVID-19 have significant differences $[5,6]$. However, we aim to show that influenza data can be used to develop a well performing model that could have been transported and used in early COVID-19 cases. The extensive external validation of the influenza developed model in early COVID-19 cases will robustly demonstrate the performance in COVID-19 patients and show areas that need adjustment and the model's limitations. The lessons learned from this study could be used to inform the development of early prediction models in future pandemics.

\section{Methods}

We performed a retrospective cohort study to develop COVID-19 prediction models for severe and critical illness. This study is reported according to the Transparent Reporting of a multivariate prediction model for Individual Prediction or Diagnosis (TRIPOD) guidelines [7].

At the start of the pandemic, there was very limited data available to develop prediction models due to the novel nature of the disease. To overcome the shortcoming of small data, we investigated whether we could use a proxy disease to develop a prediction model. This allowed 
us to utilise all available COVID-19 data for model validation. We developed models using historical data from patients with influenza or flu-like symptoms to assess a patient's individual risk of developing severe or critical illness following infection using readily available information (i.e. socio-demographics and medical history). The developed models were validated against COVID19 patients to test whether the performance transferred between the two settings.

We developed COVID-19 Estimated Risk (COVER) scores to quantify a patient's risk of hospital admission with pneumonia (COVER-H), hospitalization with pneumonia requiring intensive services or death (COVERI), or fatality (COVER-F) due to COVID-19 using the Observational Health Data Sciences and Informatics (OHDSI) Patient-Level Prediction framework [8]. The research collaboration known as OHDSI has developed standards and tools that allow patient-level prediction models to be rapidly developed and externally validated following accepted best practices [9]. This allows us to overcome two shortcomings of previous COVID19 prediction papers by reporting according to open science standards and implementing widespread external validation.

\section{Source of data}

This study used observational healthcare databases from six different countries. All datasets used in this paper were mapped into the Observational Medical Outcomes Partnership Common Data Model (OMOP-CDM) [10]. The OMOP-CDM was developed for researchers to have diverse datasets in a consistent structure and vocabulary. This enables analysis code and software to be shared among researchers, which facilitates replication and external validation of the prediction models.

The OMOP-CDM datasets used in this paper are listed in Table 1. All COVID-19 data was collected prior to $4 / 27 / 2020$.

\section{Participants}

For model development, we identified patients aged 18 or older with a general practice (GP), emergency room (ER), or outpatient (OP) visit with influenza or flu-like symptoms (fever and either cough, shortness of breath,

Table 1 Data sources formatted to the Observational Medical Outcomes Partnership Common Data Model (OMOP-CDM) used in this research (data type: claims, electronic health/medical records (EHR/EMR), general practitioner (GP))

\begin{tabular}{|c|c|c|c|c|c|}
\hline Database & $\begin{array}{l}\text { Database } \\
\text { Acronym }\end{array}$ & Country & Data type & $\begin{array}{l}\text { Contains } \\
\text { COVID-19 } \\
\text { data? }\end{array}$ & Time period \\
\hline Columbia University Irving Medical Center Data Warehouse & CUIMC & United States & EMR & Yes & $\begin{array}{l}\text { Influenza: 1990-2020 } \\
\text { COVID-19: March-April } 2020\end{array}$ \\
\hline Health Insurance and Review Assessment & HIRA & South Korea & Claims & Yes & $\begin{array}{l}\text { COVID-19: } 1^{\text {st }} \text { January- } \\
4^{\text {th }} \text { April } 2020\end{array}$ \\
\hline The Information System for Research in Primary Care & SIDIAP & Spain & $\begin{array}{l}\text { GP and } \\
\text { hospital } \\
\text { admission } \\
\text { EHRs linked }\end{array}$ & Yes & $\begin{array}{l}\text { Influenza: 2006-2017 } \\
\text { COVID-19: March } 2020\end{array}$ \\
\hline Tufts Research Data Warehouse & TRDW & United States & EMR & Yes & $\begin{array}{l}\text { Influenza: 2006-2020 } \\
\text { COVID-19: March } 2020\end{array}$ \\
\hline Department of Veterans Affairs & VA & United States & EMR & Yes & $\begin{array}{l}\text { Influenza: 2009-2010, } \\
\text { 2014-2019 } \\
\text { COVID-19: } 1^{\text {st }} \text { March- } \\
\text { 20 th April }\end{array}$ \\
\hline Optum( De-Identified ClinFormatics ${ }^{\circledR}$ Data Mart Database ${ }^{a}$ & ClinFormatics & United States & Claims & No & $2000-2018$ \\
\hline Ajou University School of Medicine Database & AUSOM & South Korea & $\mathrm{EHR}$ & No & $1996-2018$ \\
\hline Australian Electronic Practice based Research Network & AU-ePBRN & Australia & $\begin{array}{l}\text { GP and } \\
\text { hospital } \\
\text { admission } \\
\text { EHRs linked }\end{array}$ & No & 2012-2019 \\
\hline IBM MarketScan ${ }^{\circledR}$ Commercial Database & CCAE & United States & Claims & No & $2000-2018$ \\
\hline Integrated Primary Care Information & $\mathrm{IPCl}$ & Netherlands & GP & Yes & $2006-2020$ \\
\hline Japan Medical Data Center & JMDC & Japan & Claims & No & $2005-2018$ \\
\hline IBM MarketScan ${ }^{\circledR}$ Multi-State Medicaid Database & MDCD & United States & Claims & No & $2006-2017$ \\
\hline IBM MarketScan ${ }^{\circledR}$ Medicare Supplemental Database & MDCR & United States & Claims & No & $2000-2018$ \\
\hline Optum $^{\odot}$ de-identified Electronic Health Record Dataset & Optum EHR & United States & EHR & No & 2006-2018 \\
\hline
\end{tabular}

${ }^{\text {a }}$ Development database 
myalgia, malaise, or fatigue), at least 365 days of prior observation time, and no symptoms in the preceding 60 days. The initial healthcare provider interaction was used as index date, which is the point in time a patient enters the cohort.

For validation in COVID-19 we used a cohort of patients presenting at an initial healthcare provider interaction with a GP, ER, or OP visit with COVID-19 disease. COVID-19 disease was identified by a diagnosis code for COVID-19 or a positive test for the SARS-COV-2 virus that was recorded after $1 / 1 / 2020$. We required patients to be aged 18 or over, have at least 365 days of observation time prior to the index date and no diagnosis of influenza, flu-like symptoms, or pneumonia in the preceding 60 days.

\section{Outcome}

We investigated three outcomes: 1) hospitalization with pneumonia from index up to 30 days after index, 2) hospitalization with pneumonia that required intensive services (ventilation, intubation, tracheotomy, or extracorporeal membrane oxygenation) or death after hospitalization with pneumonia from index up to 30 days after are thus effectively imputed as zero, exceptions are age and sex, which are always recorded in the OMOP-CDM. In total, we derived 31,917 candidate predictors indicating the presence of unique conditions/drugs recorded prior to the index date (GP, ER, or OP visit) for each patient. When using a data-driven approach to model development, generally the resulting models contain many predictors. This may optimise performance, but can be a barrier to clinical implementation. The utility of models for COVID19 requires that they can be widely implemented across worldwide healthcare settings. Therefore, in addition to a data-driven model, we investigated two models that include fewer candidate predictors.

The age/sex model used only age groups and sex as candidate predictors. The COVER scores used a reduced set of variables, which were obtained by the following process:

1. Multiple clinicians inspected the data-driven model to identify variables that had a high standardized mean difference between patients with and without the outcome calculated using the following equation

$$
\left(\text { standardisedMeanDifference }=\frac{\text { mean with outcome }- \text { mean without outcome }}{\sqrt{\text { variance with outcome }+ \text { variance without outcome }}}\right) .
$$

index, and 3) death from index up to 30 days after index. Note that death is included in the second outcome to avoid incorrectly classifying patients who died without receiving intensive services as "low risk".

The analysis code used to construct the participant cohorts and outcomes used for development and validation can be found in the R packages located at: https:// github.com/ohdsi-studies/Covid19PredictionStudies

\section{Sensitivity analyses}

We performed sensitivity analyses which involved using different versions of the COVID-19 cohort with varying sensitivities and specificities. At the beginning of the pandemic less testing capacity was available and as such we wanted to try broader definitions. Hence, we investigated three additional definitions where we included patients with symptoms, influenza, and visits any time prior to 2020. We then performed identical analysis with these changed cohorts.

\section{Predictors}

We developed a data-driven model using age in groups $(18-19,20-25,26-30, \ldots, 95+)$, sex, and binary variables indicating the presence or absence of recorded conditions and drugs any time prior to the index date. Missing records
There are often multiple predictors which are related and correlated selected by the model, for example a model might select a condition occurrence in different time periods predating the index date. This could be simplified to one predictor saying only "Patient had condition X in history", instead of having multiple predictors specifying in which time period the condition occurred. Likewise, multiple codes that are probably related to a specific condition could be simplified in one predictor. We identified general categories from these such as 'heart disease' and 'diabetes'.

2. Phenotype definitions for each category were created. This was performed to make the definitions clinically meaningful.

3. We trained a LASSO logistic regression model on the original data using age groups, sex and the newly created predictors indicating whether the patient had any of the category predictors.

4. The coefficients of this reduced variable model were then multiplied by 10 and rounded to the nearest integer. This was done to make the model simpler to calculate.

5. This gave us the simple score-based model. 


\section{Sample size}

The models were developed using the Optum $\odot$ DeIdentified ClinFormatics ${ }^{\circledR}$ Data Mart Database. We identified 7,344,117 valid visits with influenza or flu-like symptoms, of which 4,431,867 were for patients aged 18 or older, $2,977,969$ of these had at least 365 days of prior observation time, and 2,082,277 of these had no influenza/symptoms/pneumonia in the 60 days prior to index. We selected a random sample of 150,000 patients from the total population, as research showed it is possible to efficiently develop models with near optimal performance, while reducing model complexity and computational requirements by using a sample of this size [11]. Riley et al. provide a calculator for minimum sample size, which for number of predictors $=20$, event rate $=0.05$ and $\mathrm{R}^{2}=0.1$ would require a minimum of 1698 patients [12]. This subset was used to develop the data-driven model. The full set of 2,082,077 patients was then used for the development and validation of the simple model. A small subset of this data was used to develop the data-driven model and so the presented internal performance could be optimistic. In theory this is a limitation, but it has no effect on the evidence of the external validation. Fig. 1 is a flow chart demonstrating the above exclusions and flow of data through the study.

\section{Missing data}

Age and sex are required by the OMOP-CDM used by OHDSI and will never be missing. For each condition or drug we considered no records in the database to mean the patient does not have the condition or does not receive the drug. This could lead to misclassification of patients if a patient's illness is not recorded in the database.

\section{Statistical analysis methods}

Model development followed a previously validated and published framework for the creation and validation

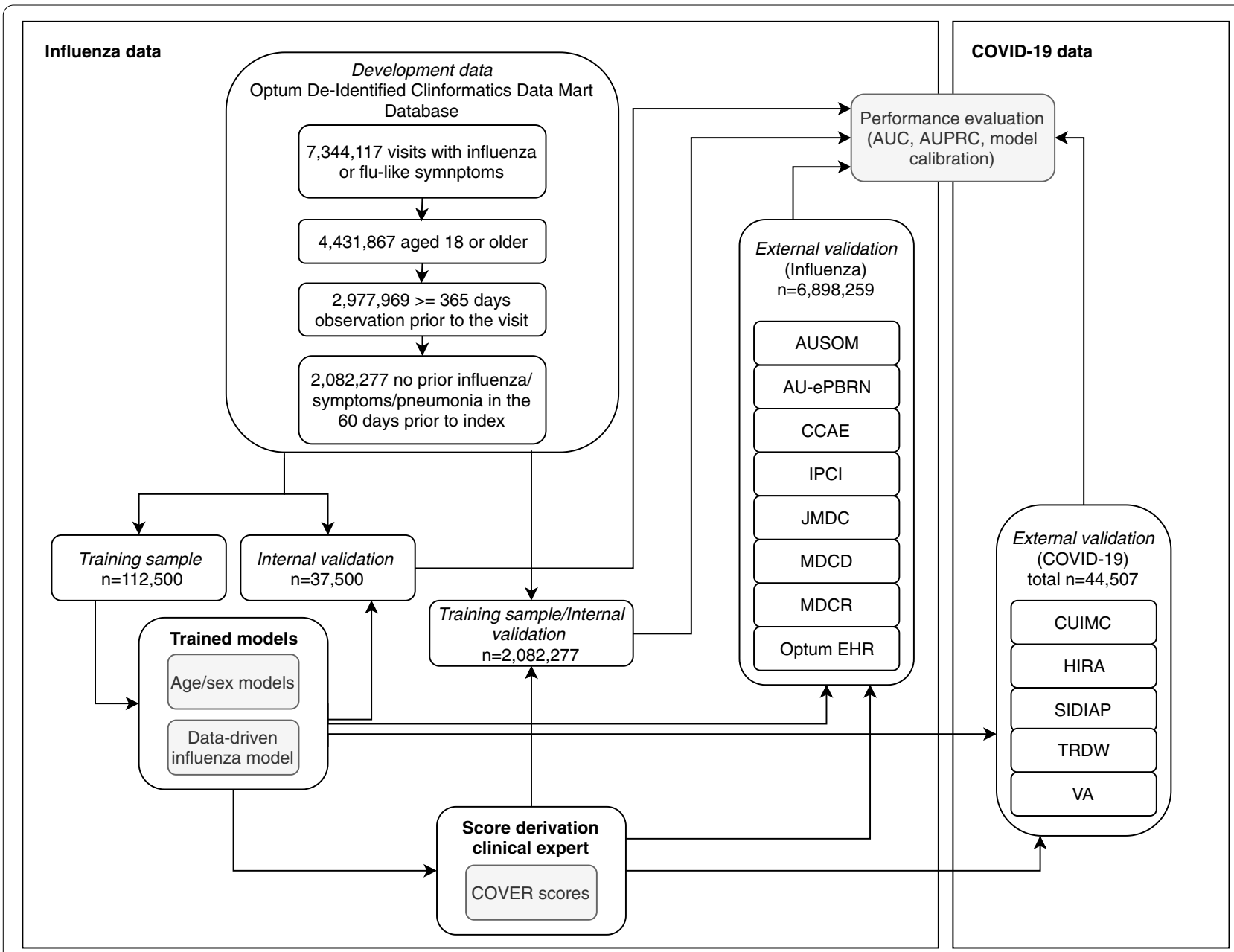

Fig. 1 A Flow chart representing the path of data in the study. This details the splits used internally for model development, the steps taken for model parsimonisation and validation and external validation 
of patient-level prediction models [8]. We used a person 'train-test split' method to perform internal validation. In the development cohort, a random split sample ('training sample') containing $75 \%$ of patients was used to develop the prediction models and the remaining $25 \%$ of patients ('test sample') was used to internally validate the models. We trained models using LASSO regularized logistic regression, using a 3-fold cross validation technique in the train-set to learn the optimal regularization hyperparameter through an adaptive search [13]. We used R (version 3.6.3) and the OHDSI Patient-Level Prediction package (version 3.0.16) for all statistical analyses [8].

To evaluate the performance of the developed models, we calculate the overall discrimination of the model using the area under the receiver operating characteristic curve (AUC), the area under the precision recall-curve (AUPRC), and the model calibration. The AUC indicates the probability that for two randomly selected patients, the patient who gets the outcome will be assigned a higher risk. The AUPRC shows the trade-off between identifying all patients who get the outcome (recall) versus incorrectly identifying patients without outcome (precision) across different risk thresholds. The model calibration is presented in a plot to examine agreement between predicted and observed risks across deciles of predicted risk. Calibration assessment is then performed visually rather than using a statistic or numeric value as this provides a better impression of the direction and scale of miscalibration [14]. Summary statistics are reported from the test samples.

We performed external validation in databases containing COVID-19 data. To do this we assessed patients with confirmed COVID-19. In addition, we performed a classical external validation in which we applied the models to identical settings across diverse patient populations with influenza or flu-like symptoms prior to 2020. We examined the external validation using AUC, AUPRC and model calibration in the same way as internally. We provide confidence intervals when the number of events is below 1000. Once the number of events increases, confidence intervals become too narrow to provide a good estimate of error.

This study adheres to open science principles for publicly prespecifying and tracking changes to study objectives, protocol, and code as described in the Book of OHDSI [15]. For transparency, the R packages for the development and external validation of the models in any database mapped to the OMOP-CDM are available on GitHub at:

https://github.com/ohdsi-studies/Covid19Predicti onStudies

\section{Results}

\section{Online results}

The complete results are available as an interactive app at: http://evidence.ohdsi.org/Covid19CoverPrediction

This application will continue to be updated as the models are validated, an archived version of the app that was released to accompany this article is available here: https://zenodo.org/record/4697417

\section{Participants}

Table 2 describes the characteristics at baseline of the patients across the databases used for development and external validation. Out of the 150,000 patients sampled with influenza or flu-like symptoms in the development database (ClinFormatics), there were 6712 patients requiring hospitalization with pneumonia, 1828 patients requiring hospitalization and intensive services with pneumonia or death, and 748 patients died within 30 days. See Table 2 for the full outcome proportions across the databases included in this study. A total of 44,507 participants with COVID-19 disease were included for external validation.

In the databases used for external validation, the patient numbers ranged from 395 (TRDW) to 3,146,743 (CCAE). The datasets had varied outcome proportions ranging from 0.06-12.47 for hospital admission, 0.014.91 for intensive services, and $0.01-12.27$ for fatality. Characteristics at baseline differed substantially between databases as can be seen in Table 2, with MDCR (a database representing retirees) containing a relatively old population of patients and a high number of comorbidities, and IPCI (a database representing general practice) showing a relatively low condition occurrence.

\section{Model performance}

The internal validation performance for each model is presented in Table 3. The external validation of the COVER scores on the COVID-19 patients is shown in Table 4. Full validation results can be seen in Appendix $1 \mathrm{~B}$ of the online supplement. Receiver operating characteristic and calibration plots are included in Fig. 2 and Appendix $1 \mathrm{C}$ of the online supplement.

\section{Model specification}

The data-driven models for hospitalization, intensive services, and fatality contained 521, 349, and 205 predictors respectively. The COVER-H, COVER-I, and COVER-F scores are presented in Fig. 3. After data-driven selection, clinicians reviewed the resulting models and created the composite predictors. This produced the COVER scores which include 7 predictors, in addition to age groups and sex, that corresponded to the following conditions 


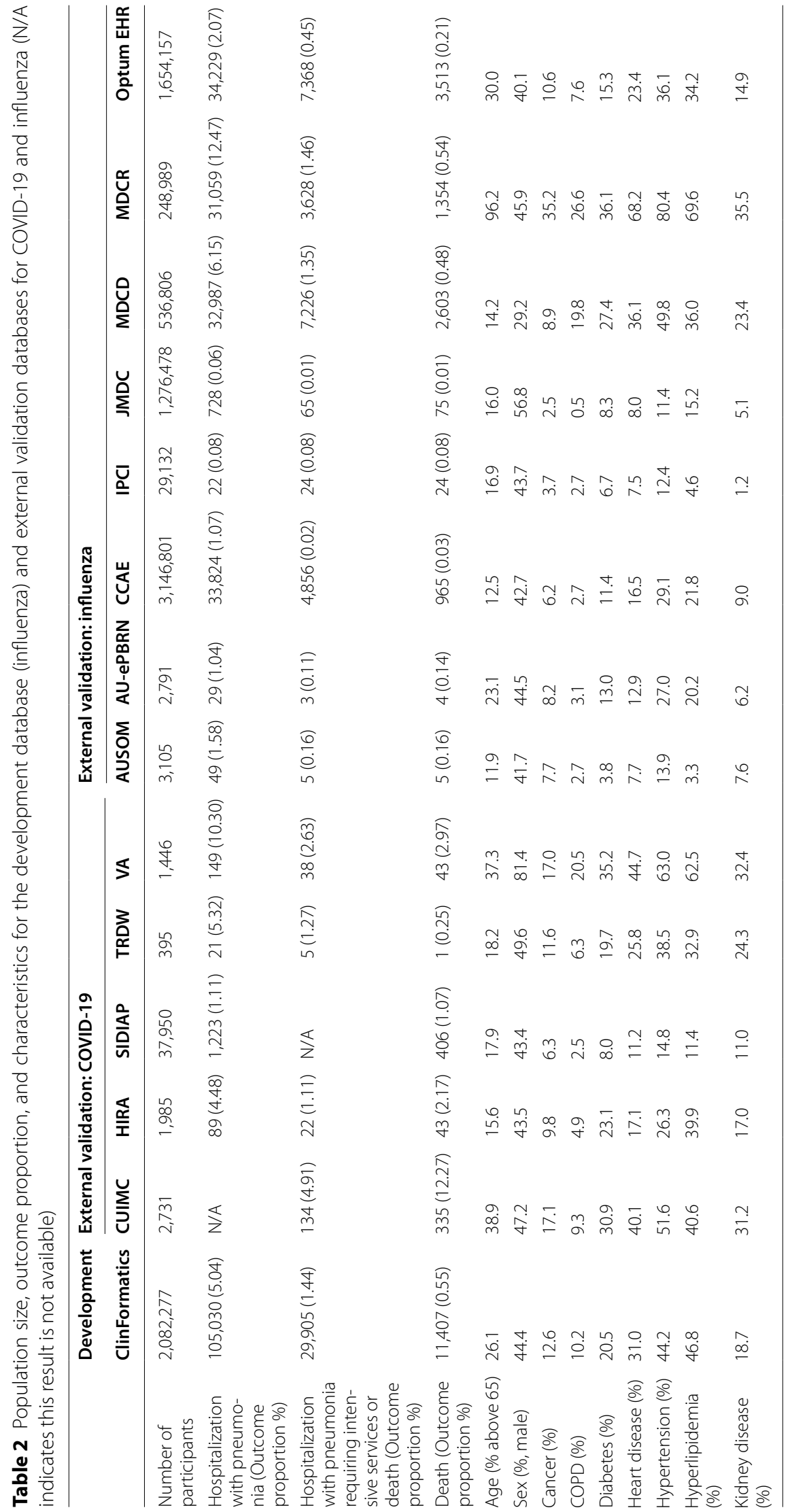


Table 3 Results for internal validation in ClinFormatics

\begin{tabular}{|c|c|c|c|c|}
\hline Outcome & Predictors & No. Variables & AUC & AUPRC \\
\hline \multirow[t]{3}{*}{ Hospitalization with pneumonia } & Conditions/drugs + age/sex & 521 & 0.852 & 0.224 \\
\hline & Age/sex & 2 & 0.818 & 0.164 \\
\hline & COVER-H & 9 & 0.840 & 0.120 \\
\hline \multirow{3}{*}{$\begin{array}{l}\text { Hospitalization with pneumonia requiring } \\
\text { intensive services or death }\end{array}$} & Conditions/drugs + age/sex & 349 & 0.860 & 0.070 \\
\hline & Age/sex & 2 & 0.821 & 0.049 \\
\hline & COVER-I & 9 & 0.839 & 0.059 \\
\hline \multirow[t]{3}{*}{ Fatality } & Conditions/drugs + age/sex & 205 & 0.926 & 0.069 \\
\hline & Age/sex & 2 & 0.909 & 0.037 \\
\hline & COVER-F & 9 & 0.896 & 0.039 \\
\hline
\end{tabular}

Table 4 Results of external validation of the COVER scores on COVID-19 patients with a GP, ER, or OP visit in $2020\left({ }^{*}\right.$ Confidence interval is not reported as the number of outcomes is larger than 1000)

\begin{tabular}{|c|c|c|c|}
\hline Outcome & Database & $\begin{array}{l}\text { AUC }(95 \% \\
\text { confidence } \\
\text { interval) }\end{array}$ & AUPRC \\
\hline \multirow{4}{*}{$\begin{array}{l}\text { Hospitalization with pneu- } \\
\text { monia } \\
\text { (COVER-H) }\end{array}$} & HIRA & $0.806(0.762-0.851)$ & 0.134 \\
\hline & SIDIAP & $0.748^{*}$ & 0.072 \\
\hline & TRDW & $0.731(0.611-0.851)$ & 0.132 \\
\hline & VA & 0.689 (0.649-0.729) & 0.179 \\
\hline \multirow{3}{*}{$\begin{array}{l}\text { Hospitalization with pneumo- } \\
\text { nia requiring intensive services } \\
\text { or death } \\
\text { (COVER-I) }\end{array}$} & CUIMC & $0.734(0.699-0.769)$ & 0.100 \\
\hline & HIRA & $0.910(0.889-0.931)$ & 0.053 \\
\hline & VA & $0.763(0.708-0.818)$ & 0.058 \\
\hline \multirow{4}{*}{$\begin{array}{l}\text { Fatality } \\
\text { (COVER-F) }\end{array}$} & CUIMC & $0.820(0.796-0.840)$ & 0.400 \\
\hline & HIRA & $0.898(0.857-0.940)$ & 0.150 \\
\hline & SIDIAP & 0.895 (0.881-0.910) & 0.083 \\
\hline & VA & $0.717(0.642-0.791)$ & 0.068 \\
\hline
\end{tabular}

existing any time prior to the index date: cancer, chronic obstructive pulmonary disease, diabetes, heart disease, hypertension, hyperlipidemia, and kidney disease (chronic and acute). A description of the covariates can be found in Appendix 1A of the online supplement. The COVER scores are detailed in Fig. 3 and are accessible online under the calculator tab at: http://evidence.ohdsi. org:3838/Covid19CoverPrediction/

Figure 3 also provides a risk converter, which allows for easy conversion between the risk score and predicted risk of the outcomes. The scores can be converted to a probability by applying the logistic function: $1 /(1+$ exp. ((risk score-93)/10)). Furthermore, we provide a plot of the probability distribution for each of the three models from patients in Clinformatics to demonstrate the expected regions the probabilities fall into. To calculate the COVER scores using Fig. 3, a clinician first needs to identify which conditions the patient has. The points for the corresponding predictors are then added to arrive at the total score. For example, if a 63-year-old female patient has diabetes and heart disease, then her risk score for hospital admission (COVER-H) is 43 (female sex $)+4$ (heart disease $)+3$ (diabetes $)+15($ age $)=65$. The risk scores for intensive services (COVER-I) and fatality (COVER-F) are 51 and 47, respectively. Using the risk converter in Fig. 3, a score of 65 corresponds to a risk of $6 \%$. Scores of 51 and 47 correspond to 1.5 and $1 \%$, respectively.

\section{Discussion \\ Interpretation}

We developed and externally validated models using large datasets of influenza patients to quantify a patient's risk of developing severe or critical illness due to COVID-19. In the development data, the 9-predictor COVID-19 Estimated Risk (COVER) scores were a good trade-off between model complexity and performance, as the AUCs were generally close to the large datadriven models. In the development database the COVER scores achieved an AUC of 0.84 when predicting which patients will be hospitalized or require intensive services and an AUC of 0.90 when predicting which patients will die within 30 days. When validated on 1985 COVID-19 patients in South Korea the COVER-H score achieved an AUC of 0.81, COVER-I and COVER-F achieved an AUC of 0.90 and 0.91. When applied to 37,950 COVID19 Spanish patients COVER-H had an AUC of 0.75 and performed better when predicting fatality (COVER-F: AUC 0.89). When applied to US patients, the COVER-I and COVER-F models achieved AUCs of 0.73 and 0.82 in CUIMC, VA performed similarly with AUCs of 0.76 and 0.72 respectively. The VA also achieved 0.69 for COVER$\mathrm{H}$. The results show reasonable performance with some inconsistency across a range of countries.

A visual assessment of calibration plots across validations showed reasonable calibration in HIRA, 


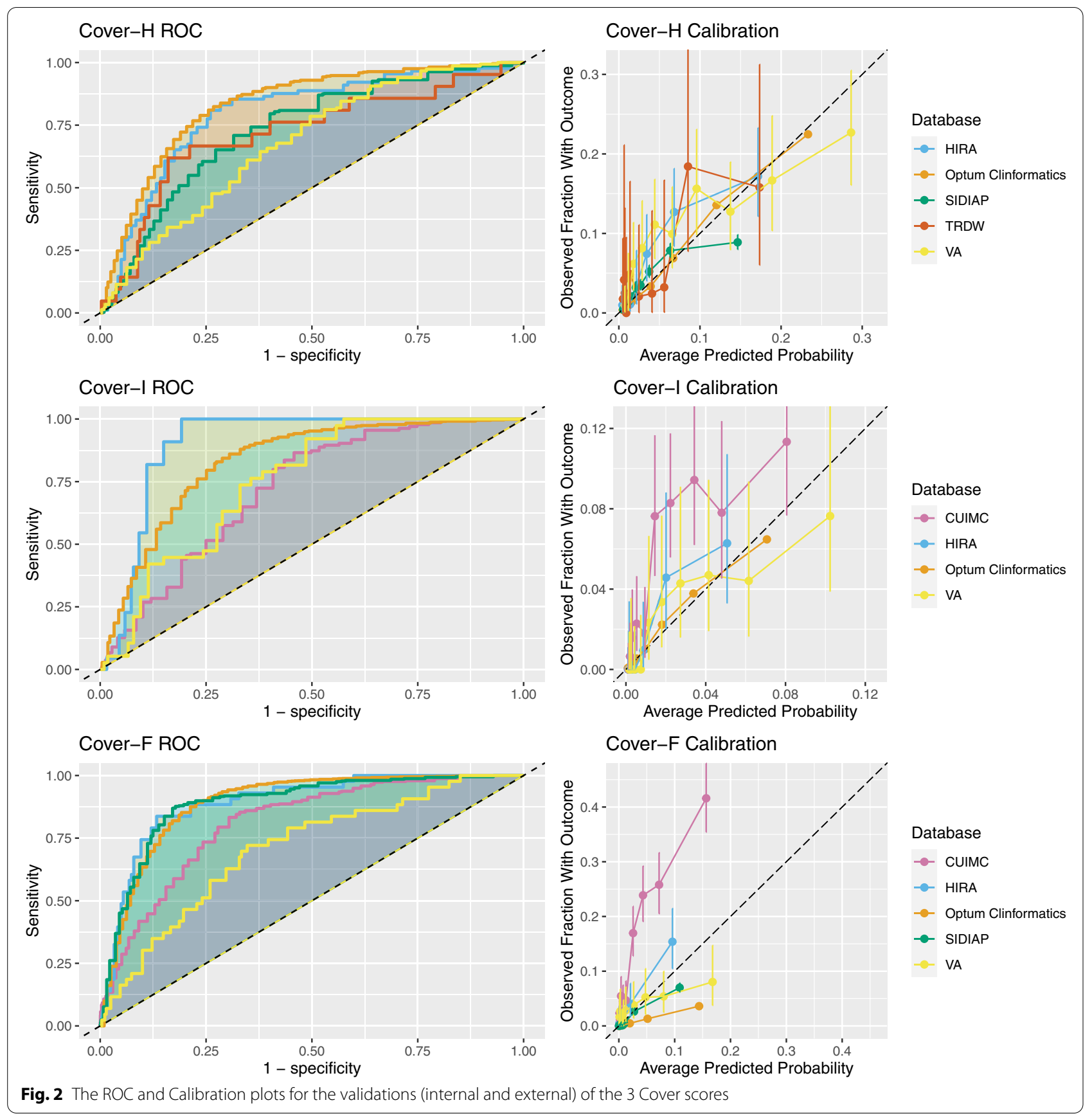

SIDIAP, and VA. There was a slight overestimation of risk amongst oldest and highest risk strata in SIDIAP, and to a lesser extent in HIRA. The calibration was poor in CUIMC, as risk was often underestimated. This may be due to CUIMC containing mostly hospitalized COVID-19 patients, so the CUIMC cohort are experiencing more severe COVID-19. The VA showed some miscalibration in the lowest and highest risk strata. The observed miscalibration is possibly due to the differing severities of the diseases used for model development and calibration. However, miscalibration could also be due to other differences in populations not caused by the use of a proxy disease. The variable calibration results suggest that the model's performance should be assessed and the model should potentially be recalibrated before being implemented in a local context. A simple method to do this is by adjusting the baseline risk based upon the differences found between 


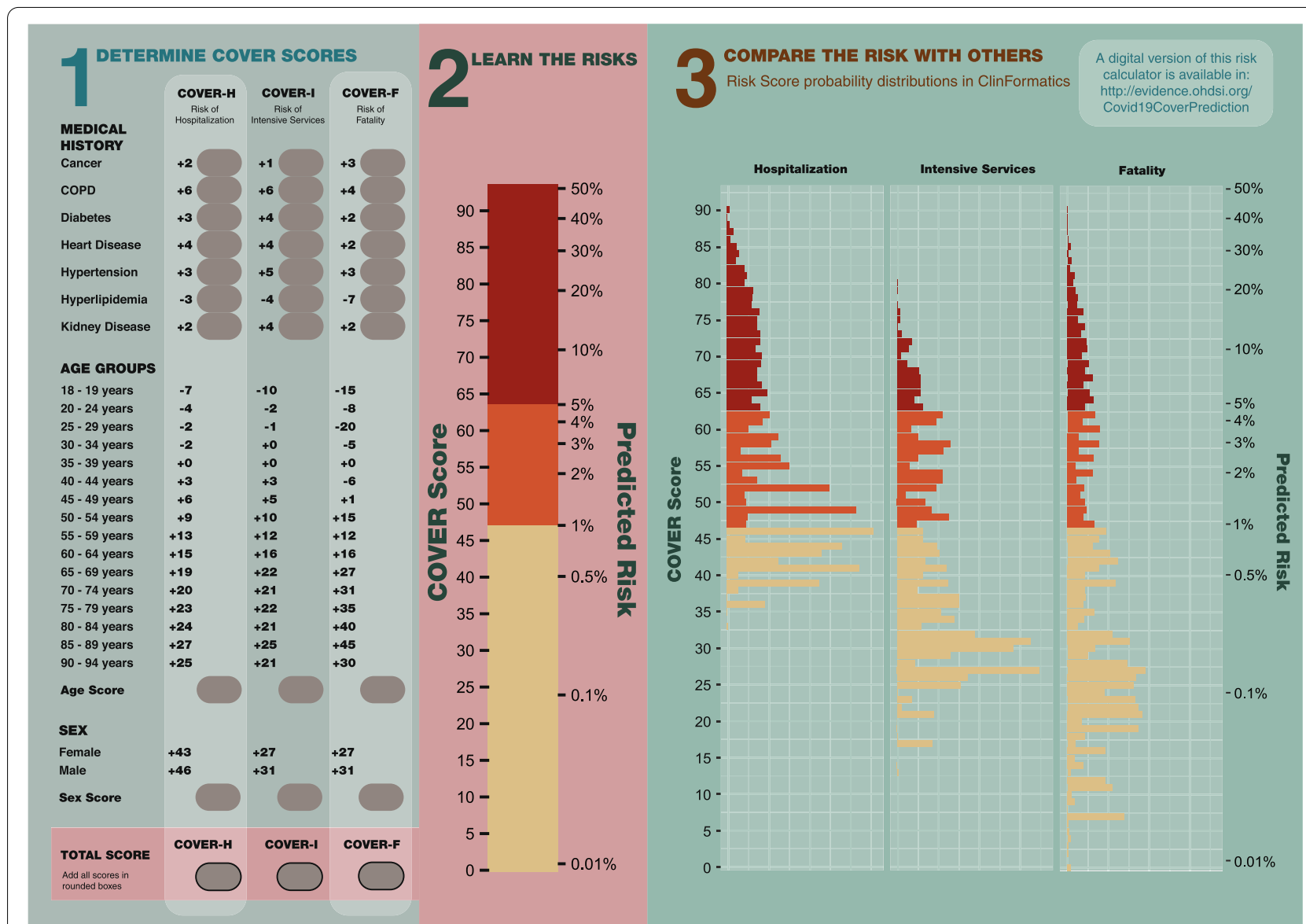

Fig. 3 A graphic showing how to calculate the 3 Cover scores with a nomogram to convert the raw score into a percentage risk. There is also a distribution of scores found using internal validation to allow for comparison of a patients score to the wider population

development and validation populations using an adjustment factor derived from the differences in case mix between development and validation settings [16, 17].

The age/sex models also show reasonable performance, and these predictors are among the main contributors to performance in the COVER scores. This suggests these models could also be suitable if access to medical history is difficult.

These results showed that training in large historical influenza data was an effective strategy to develop models for COVID-19 patients. We also performed sensitivity analyses using more sensitive COVID-19 definitions, for example including patients with symptoms, influenza, and visits any time prior to 2020 . The results did not show much deviation from the specific definition (online supplement Appendix 1B). Our results show that quantifying a symptomatic patient's risk based on a small selection of comorbidities as well as age/sex gives improved model performance.

\section{Limitations}

First, it has become clear that there are differences in the underlying nature of the two diseases, particularly in respect to the severity of symptoms in COVID-19 patients compared with influenza patients. Therefore, it is possible another disease may have provided a better proxy than influenza.

Second, despite preserving all the target disease data for validation, we still had relatively low outcome numbers. In the CUIMC, HIRA, SIDIAP, and VA COVID-19 databases we either reached or approached the threshold for reliable external validation of 100 patients who experience the outcome of interest $[18,19]$, but the results of TRDW might not be reliable.

Furthermore, the data reported early during the COVID-19 pandemic was noisy and skewed. This might cause misclassification in the target and outcome cohorts. In order to counter this, we performed sensitivity analysis using cohorts that included broad and narrow COVID-19 definitions, the impact of this on the results was minimal. 
The use of a 30-day risk window has the limitation that if a patient experiences an outcome after the time window, this will be (incorrectly) recorded as a non-event. There is further potential misclassification of predictors, for example, if a disease is incorrectly recorded in a patient's history. Moreover, the result of the phenotype generation process is not fully reproducible due to the use of clinician expertise, which is an unresolved problem in much epidemiological work. However, the phenotype development process is reproducible and the phenotypes generated are provided. The evidence in the paper shows the models to be robust and transportable.

We were unable to include some suspected disease predictors in the analysis as these are not readily available (e.g. lymphocyte count, lung imaging features) or inconsistently collected and reported across the various databases included in the study (e.g. BMI, ethnicity). However, due to the high load on healthcare systems and the contagious nature of the disease we believe it is useful to have a model that does not require a patient to be either in hospital or another setting to receive tests. A similar issue also meant we were not able to validate the COVER-H score in CUIMC (it mostly contains ER or hospitalized COVID-19 patients) and the COVER-I score in SIDIAP (due to a lack of information on intensive services in the database).

Finally, concerns exist over the clinical validity of claims data, however we were able to develop models using claims data that transported well into EHR data. There is the potential for some overlap of patients between claims and EHR databases, although this number is likely to be small.

\section{Implications}

The results show we were able to develop models that use historical influenza patient's socio-demographics and medical history to predict their risk of becoming severely or critically ill when infected with COVID-19. To our knowledge, this is the first study that has been able to extensively externally validate prediction models on COVID-19 patients at a global scale. The adequate performance of the COVER scores in COVID-19 patients (as quantified by consistent finding of AUC $>0.7$ in new settings) show these scores could have been used to identify patients who should have been shielded from COVID-19 in the early stages of the pandemic.

\section{Conclusion}

In this paper we developed and validated models that can predict which patients presenting with COVID-19 are at high risk of experiencing severe or critical illness. This research demonstrates that it is possible to develop a prediction model rapidly using historical data of a similar disease that, once re-calibrated with contemporary data and outcomes from the current outbreak, could be used to help inform strategic planning and healthcare decisions.

\section{Supplementary Information}

The online version contains supplementary material available at https://doi. org/10.1186/s12874-022-01505-z.

\section{Additional file 1.}

Additional file 2.

\section{Acknowledgements}

The authors would like to thank the OHDSI community for their contributions to the tools used for this analysis.

The authors appreciate healthcare professionals dedicated to treating COVID-19 patients in Korea, and the Ministry of Health and Welfare and the Health Insurance Review \& Assessment Service of Korea for sharing invaluable national health insurance claims data in a prompt manner.

\section{Authors' contributions}

All authors made substantial contributions to the conception or design of the work; JMR and RDW led the data analysis; all authors were involved in the analysis and interpretation of data for the work; all authors have contributed to the drafting and revising critically the manuscript for important intellectual content; all authors have given final approval and agree to be accountable for all aspects of the work.

\section{Funding}

This project has received support from the European Health Data and Evidence Network (EHDEN) project. EHDEN received funding from the Innovative Medicines Initiative 2 Joint Undertaking (JU) under grant agreement No 806968. The JU receives support from the European Union's Horizon 2020 research and innovation programme and EFPIA.

This project is funded by the Health Department from the Generalitat de Catalunya with a grant for research projects on SARS-CoV-2 and COVID-19 disease organized by the Direcció General de Recerca i Innovació en Salut.

The University of Oxford received a grant related to this work from the Bill \& Melinda Gates Foundation (Investment ID INV-016201), and partial support from the UK National Institute for Health Research (NIHR) Oxford Biomedical Research Centre.

DPA is funded through a NIHR Senior Research Fellowship (Grant number SRF-2018-11-ST2-004). The views expressed in this publication are those of the author(s) and not necessarily those of the NHS, the National Institute for Health Research, or the Department of Health.

AP- $U$ is supported by Fundacion Alfonso Martin Escudero and the Medical Research Council (grant numbers MR/K501256/1, MR/N013468/1). BSKH is funded through Innovation Fund Denmark (5153-00002B) and the Novo Nordisk Foundation (NNF14CC0001).

This work was also supported by the Bio Industrial Strategic Technology Development Program (20001234) funded by the Ministry of Trade, Industry \& Energy (MOTIE, Korea) and a grant from the Korea Health Technology R\&D Project through the Korea Health Industry Development Institute (KHIDI), funded by the Ministry of Health \& Welfare, Republic of Korea [grant number: HI16C0992].

This project is part funded by the UNSW RIS grant.

This research received funding support from the US Department of Veterans Affairs and the VA Informatics and Computing Infrastructure (VA HSR RES 13-457). The views and opinions expressed are those of the authors and do not necessarily reflect those of the Department of Veterans Affairs or the United States Government.

\section{Availability of data and materials}

The datasets generated and/or analysed during the current study are not publicly available due to patient privacy and data protection concerns. 
Information on access to the databases is available from the corresponding author.

\section{Declarations}

\section{Ethics approval and consent to participate}

The manuscript uses secondary data and as such no human participants were involved in the study and informed consent was not necessary at any site.

\section{Consent for publication}

Not applicable.

\section{Competing interests}

RDW, AM, CY, TDS, TF, JJ, CK, YR, AW, AA, MHO, MA, CA, EB, YHC, ID, MTFA, SFB, JAK, STL, FN, RWP, NP, TS, MS, EWS, SCY, LZ(Zhang), LZ(Zhou), report no conflicts of interest.

MM reports grants from Department of Veteran Affairs, grants from National Institutes of Health, outside the submitted work; SLD reports grants from Anolinx, LLC, grants from Astellas Pharma, Inc., grants from AstraZeneca Pharmaceuticals $\mathrm{LP}$, grants from Boehringer Ingelheim International $\mathrm{GmbH}$, grants from Celgene Corporation, grants from Eli Lilly and Company, grants from Genentech Inc., grants from Genomic Health, Inc., grants from Gilead Sciences Inc., grants from GlaxoSmithKline PLC, grants from Innocrin Pharmaceuticals Inc., grants from Janssen Pharmaceuticals, Inc., grants from Kantar Health, grants from Myriad Genetic Laboratories, Inc., grants from Novartis International AG, grants from Parexel International Corporation, outside the submitted work; BK-H reports grants from Innovation Fund Denmark, grants from Novo Nordisk Foundation, during the conduct of the study; DPA reports grants and other from UCB Biopharma SRL, grants and other from Amgen, grants from Servier Laboratoires, outside the submitted work; DM reports grants from Wellcome Trust (Clinical Research Development Fellowship), grants from Chief Scientist Office (CSO), grants from Health Data Research UK (HDR-UK), grants from National Institute of Health Research (NIHR), outside the submitted work; AP-U reports grants from Fundacion Alfonso Martin Escudero, grants from Medical Research Council, outside the submitted work; GH reports grants from NIH National Library of Medicine, during the conduct of the study; grants from Janssen Research, outside the submitted work; JMR, PBR, AS, GR are employees of Janssen Research and Development and are shareholders of Johnson \& Johnson. KK and CR report as employees of IQVIA. MR reports personal fees from Bayer Hispania, S.L., outside the submitted work; PLK and LZ are AbbVie employees. MAS reports grants from US National Institutes of Health, grants from IQVIA, personal fees from Janssen Research and Development, personal fees from Private Health Management, during the conduct of the study; PRR reports grants from Innovative Medicines Initiative, grants from Janssen Research and Development, during the conduct of the study; GM is a full-time employee of Janssen Latin America, outside the submitted work.

\section{Author details}

'Department of Medical Informatics, Erasmus University Medical Center, Doctor Molewaterplein, 403015, GD, Rotterdam, The Netherlands. ${ }^{2}$ Fundacio Institut Universitari per a la recerca a l'Atencio Primaria de Salut Jordi Gol i Gurina (IDIAPJGol), Barcelona, Spain. ${ }^{3}$ Department of Veterans Affairs, University of Utah, Salt Lake City, UT, USA. ${ }^{4}$ Department of Biomedical Informatics, Columbia University, New York, NY, USA. ${ }^{5}$ School of Public Health and Community Medicine, UNSW, Sydney, Australia. ${ }^{6}$ Department of Biomedical Sciences, Ajou University Graduate School of Medicine, Suwon, Republic of Korea. ${ }^{7}$ Department of Big Data Strategy, National Health Insurance Service, Wonju, Republic of Korea. ${ }^{8}$ Tufts University School of Medicine, Institute for Clinical Research and Health Policy Studies, Boston, MA 02111, USA. ${ }^{9}$ Independent Epidemiologist, OHDSI, Rotterdam, The Netherlands. ${ }^{10}$ So Ahn Public Health Center, Wando County Health Center and Hospital, Wando, Republic of Korea. ${ }^{11}$ Nuffield Department of Clinical Neurosciences, University of Oxford, Oxford, UK. ${ }^{12}$ Centre for Statistics in Medicine, NDORMS, University of Oxford, Oxford, UK. ${ }^{13}$ Department of Infectious Diseases, Ajou University School of Medicine, Suwon, Republic of Korea. ${ }^{14}$ Center for Surgical Science, Koege, Denmark. ${ }^{15}$ Faculty of Medicine, University of Sao Paulo, Sao Paulo, Brazil. ${ }^{16} \mathrm{Clinical}$ Pharmacology Unit, Zealand University Hospital, Roskilde, Denmark. ${ }^{17}$ NNF Centre for Protein Research, University of Copenhagen, Copenhagen, Denmark.

${ }^{18}$ Abbvie, Chicago, USA. ${ }^{19}$ Real World Solutions, IQVIA, Cambridge, MA, USA.

${ }^{20}$ Janssen Latin America, Buenos Aires, Argentina. ${ }^{21}$ Department of Veterans
Affairs, Washington D. C, USA. ${ }^{22}$ Vanderbilt University, Nashville, USA. ${ }^{23}$ Division of Population Health and Genomics, University of Dundee, Dundee, UK. ${ }^{24} \mathrm{School}$ of Public Health and Community Medicine, Institute of Medicine, Sahlgrenska Academy, University of Gothenburg, Gothenburg, Sweden. ${ }^{25}$ Department of Biomedical Informatics, Ajou University School of Medicine, Suwon, Republic of Korea. ${ }^{26}$ Quality Use of Medicines and Pharmacy Research Centre, University of South Australia, Adelaide, Australia. ${ }^{27}$ Janssen Research \& Development, Titusville, NJ, USA. ${ }^{28}$ Bayer Pharmaceuticals, Bayer Hispania, S.L., Barcelona, Spain. ${ }^{29}$ Department of Public Health, Erasmus University Medical Center, Rotterdam, The Netherlands. ${ }^{30}$ Department of Biomedical Data Sciences, Leiden University Medical Center, Leiden, The Netherlands. ${ }^{31}$ Department of Biostatistics, UCLA Fielding School of Public Health, University of California, Los Angeles, CA, USA. ${ }^{32}$ School of Public Health, Peking Union Medical College, Beijing, China. ${ }^{33}$ Melbourne School of Public Health, The University of Melbourne, Melbourne, Victoria, Australia.

Received: 6 January 2021 Accepted: 3 January 2022

Published online: 30 January 2022

\section{References}

1. World Health Organization. Clinical management of severe acute respiratory infection (SARI) when COVID-19 disease is suspected: interim guidance, 13 march 2020. Geneva: World Health Organization; 2020.

2. Prieto-Alhambra D, Ballo E, Coma-Redon E, et al. Hospitalization and 30-day fatality in 121,263 COVID-19 outpatient cases. medRxiv. 2020:2020.2005.2004.20090050.

3. World Health Organization. Coronavirus disease 2019 (COVID-19) situation report - 51 2020, 11 march 2020. Geneva: World Health Organization; 2020.

4. Wynants L, Van Calster B, Bonten MMJ, et al. Prediction models for diagnosis and prognosis of covid-19 infection: systematic review and critical appraisal. BMJ. 2020;369:m1328.

5. Piroth $L$, Cottenet J, Mariet AS, et al. Comparison of the characteristics, morbidity, and mortality of COVID-19 and seasonal influenza: a nationwide, population-based retrospective cohort study. Lancet Respir Med. 2021;9(3):251-9.

6. Petersen E. COVID-19 is not influenza. Lancet Respir Med. 2021:9(3):219-20.

7. Moons KG, Altman DG, Reitsma JB, et al. Transparent reporting of a multivariable prediction model for individual prognosis or diagnosis (TRIPOD): explanation and elaboration. Ann Intern Med. 2015;162(1):W1-73.

8. Reps JM, Schuemie MJ, Suchard MA, Ryan PB, Rijnbeek PR. Design and implementation of a standardized framework to generate and evaluate patient-level prediction models using observational healthcare data. J Am Med Inform Assoc. 2018;25(8):969-75.

9. Reps JM, Williams RD, You SC, et al. Feasibility and evaluation of a largescale external validation approach for patient-level prediction in an international data network: validation of models predicting stroke in female patients newly diagnosed with atrial fibrillation. BMC Med Res Methodol. 2020;20(1):102.

10. Overhage JM, Ryan PB, Reich CG, Hartzema AG, Stang PE. Validation of a common data model for active safety surveillance research. J Am Med Inform Assoc. 2012;19(1):54-60.

11. John LH, Kors JA, Reps JM, Ryan PB, Rijnbeek PR. How little data do we need for patient-level prediction? arXiv preprint arXiv:200807361; 2020.

12. Riley RD, Ensor J, Snell KIE, et al. Calculating the sample size required for developing a clinical prediction model. BMJ. 2020;368:m441.

13. Suchard MA, Simpson SE, Zorych I, Ryan P, Madigan D. Massive parallelization of serial inference algorithms for a complex generalized linear model. ACM Trans Model Comput Simul (TOMACS). 2013;23(1):1-17.

14. Steyerberg EW, Vergouwe Y. Towards better clinical prediction models: seven steps for development and an ABCD for validation. Eur Heart $\mathrm{J}$. 2014;35(29):1925-31.

15. Observational health data sciences and informatics. The Book of OHDSI. OHDSI; 2019.

16. Steyerberg EW, Borsboom GJ, van Houwelingen HC, Eijkemans MJ, Habbema JD. Validation and updating of predictive logistic regression models: a study on sample size and shrinkage. Stat Med. 2004;23(16):2567-86. 
17. Janssen KJ, Moons KG, Kalkman CJ, Grobbee DE, Vergouwe Y. Updating methods improved the performance of a clinical prediction model in new patients. J Clin Epidemiol. 2008;61(1):76-86.

18. Vergouwe Y, Steyerberg EW, Eijkemans MJ, Habbema JDF. Substantial effective sample sizes were required for external validation studies of predictive logistic regression models. J Clin Epidemiol. 2005;58(5):475-83.

19. Collins GS, Ogundimu EO, Altman DG. Sample size considerations for the external validation of a multivariable prognostic model: a resampling study. Stat Med. 2016;35(2):214-26.

\section{Publisher's Note}

Springer Nature remains neutral with regard to jurisdictional claims in published maps and institutional affiliations.

- fast, convenient online submission

- thorough peer review by experienced researchers in your field

- rapid publication on acceptance

- support for research data, including large and complex data types

- gold Open Access which fosters wider collaboration and increased citations

- maximum visibility for your research: over 100M website views per year

At BMC, research is always in progress.

Learn more biomedcentral.com/submissions 